\title{
Neuropsychiatry and COVID-19: An Overview
}

Marcos Altable, Juan Moisés de la Serna

Funding: The author(s) received no specific funding for this work.

Potential competing interests: The author(s) declared that no potential competing interests exist.

\begin{abstract}
The studies that exist so far to the current pandemic of COVID-19 produced by the SARS-CoV-2 coronavirus, report neuropsychiatric symptoms only as part of the manifestation of the disease in its terminal phase. However, even if the infection is mild, moderate or severe, there are neuropsychiatric symptoms since the beginning of the disease. Already in the advanced stages of the severe acute respiratory syndrome (SARS), different neuropsychiatric manifestations have been reported as a form of clinical expression of the course of infection, which could be a direct output of cerebral hypoxia due to respiratory failure, viral brain tissue infection or encephalitis, immune system reaction or cytokine storm or a combination of all these factors.

Neuropsychiatric manifestations include anxiety symptoms, panic attacks, depression, mental confusion, acute confusional syndrome, psychomotor excitement, psychosis, and even suicidal tendencies
\end{abstract}

Introduction

The studies that exist so far to the current pandemic of COVID-19 produced by the SARS-CoV-2 coronavirus, report neuropsychiatric symptoms only as part of the manifestation of the disease in its terminal phase. However, even if the infection is mild, moderate or severe, there are neuropsychiatric symptoms since the beginning of the disease. Already in the advanced stages of the severe acute respiratory syndrome (SARS), different neuropsychiatric manifestations have been reported as a form of clinical expression of the course of infection, which could be a direct output of cerebral hypoxia due to respiratory failure, viral brain tissue infection or encephalitis, immune system reaction or cytokine storm or a combination of all these factors. Neuropsychiatric manifestations include anxiety symptoms, panic attacks, depression, mental confusion, acute confusional syndrome, psychomotor excitement, psychosis, and even suicidal tendencies.[1] 
According to studies reviewed to date, neuropsychiatric symptoms can be divided into symptoms of the central nervous system (CNS), such as headache, dizziness, vertigo, altered consciousness, confusion, ataxia, acute cerebrovascular disease and seizures; peripheral nervous system (PNS) symptoms, such as anosmia, dysgeusia, neuralgia, and diarrhoea; and psychiatric symptoms such as apathy, depression, anorexia, psychosis, acute confusional syndrome, and ag itation.

Several investigations have indicated a direct relationship between chronic diseases such as human immunodeficiency virus (HIV) and tuberculosis, with mental disorders such as depression [2,3], in the general population [4,5]. Similarly, studies conducted during and after epidemics such as SARS 2003 and Ebola 2014, found that there was a widespread behaviour induced by the hyperactive reaction among the general public $[6,7]$. In addition to this, various psychiatric disorders such as anxiety, depression, and post-

traumatic stress disorder (PTSD) were found mainly in survivors and healthcare workers [8-10].

Previous studies have reported adverse psychological reactions to the 2003 SARS outbreak among healthcare workers.[11-14]. Studies showed that these health workers feared contagion and infection from their family, friends and colleagues [14], felt uncertainty and stig matization [13,14], reported aversion to work [13], and high levels of stress, anxiety and symptoms of depression [12], that could have long-term psychological implications [12]. Similar concerns are now emerging about mental health, psychological adjustment, and recovery for both the ill and the health workers who treat and care for patients with COVID-19. Besides, as the WHO has highlighted in its state of mental health, stigmatization and the scapegoat of affected people, including health professionals, is very common during epidemics $[15,16]$. Unfortunately, this trend continues to prevail during the current COVID-19 outbreak, as many people of Asian origin, explicitly Chinese, are victims of xenophobia and social stigmatization, with high levels of threats online and during public interactions [17].

Aggression, frustration and post-traumatic stress disorder Initially, the public emotional response to any pandemic is one of extreme fear and uncertainty that generally leads to neg ative social behaviours and can involve public mental health problems such as anxiety, insomnia, aggression, depression, frustration, and hysteria [15]. Compared to previous studies related to SARS outbreaks, patients with COVID-19 (defined or suspected), and in quarantine, are more likely to suffer from loneliness, anger and frustration [18]. Similarly, another concern is that survivors and mental health professionals suffer from post-traumatic rhinitis disorder (PTSD). In a study conducted at a Beijing hospital, quarantined health workers who worked in high-risk 
settings or had a family member with SARS reported a significantly higher level of posttraumatic stress symptoms compared to those without similar experiences [19-21]. Similarly, medical professionals who dealt with SARS patients or worked during the SARS outbreak also reported fear, anxiety, depression, and frustration $[1,20]$. In comparison, health professionals working in COVID-19 quarantined units, lacking adequate protective measures and suffering the death of other physicians, may develop severe symptoms of post-traumatic stress disorder. For this reason, mental health professionals must apply therapies such as the stress adaptation model to reduce the level of high stress in this population [22].

Anxiety and obsessive-compulsive disorder Any situation of uncertainty can lead to alarmist behaviour, and with the influence of COVID-19, confinement, and the absence of adequate treatment for infectious disease, this situation has led the masses to moments of panic and anxiety [23]. Similarly, the obsession with contamination, which is persistent, for cleaning and the constant need for washing or sterilization is generally classified under obsessive-compulsive disorder [24]. Moreover, in the event of a pandemic caused by an infectious virus, this disorder can contribute to panic, resulting in disruption and impairment of the necessary daily activities of people. Mental health professionals and clinical psychologists must be alert to these impending problems and try to resolve them with the utmost care.

\section{Pathophysiology}

In many patients infected with SARS-CoV-2 and, even more, in those who develop moderate to severe respiratory failure, neuropsychiatric disorders can occur due to different mechanisms that can act concomitantly, such as cerebral hypoxia due to respiratory failure (confusion mental), cytokine storm due to exaggerated immune response (apathy, anorexia and muscular pain) and encephalitis due to direct brain infection (agitation and psychosis) $[9,25,26]$.

Recently, the angiotensin 2-converting enzyme (ACE2) has been identified as the functional receptor for SARS-CoV-2, which is present in multiple human organs, including the nervous system and the musculoskeletal system. The expression and distribution of ACE2 explain why SARS-CoV-2 can produce neuropsychiatric symptoms through direct and indirect mechanisms. Coronavirus encephalitis has also been confirmed, as in SARSCoV and MERS-CoV. The researchers detected SARS-CoV nucleic acid in the cerebrospinal fluid of these patients and also brain tissue from the autopsies [11-13]. Another mechanism involved in many genetically predisposed patients is the cytokine storm generated by the interaction of the immune system with the virus, which, in 
addition to the systemic repercussion, primarily affects the nervous system. Cytokine storm resembles macrophage activation syndrome (MAS), a severe condition that presents with hypercytokinemia, fever, cytopenia and hyperferritinemia; pulmonary involvement (including ARDS) and is associated with the severity of COVID-19 disease. It is characterized by an increase in interleukin (IL) -2, IL-7, granulocyte colony-stimulating factor, interferon-inducible protein $10-\gamma$, monocyte chemoattractant protein 1 , macrophage inflammatory protein $1-\alpha$ and tumor necrosis factor- $\alpha$ [27].. Brain cytokines produce behavioural changes during the course of an illness or infection, manifesting depressive symptoms such as emotional hyperresponsiveness, apathetic syndrome, anhedonia, hyporexia, weight loss, hypersomnia, alteration of the circadian rhythm, fatigue and chronic pain, psychomotor inhibition, demotivation, disinterest and alteration of higher mental functions, etc. It would appear that during acute coronavirus infection, immune hyperreactivity generates this behaviour syndrome with such variable neuropsychiatric symptoms. This is important since in terms of COVID-19 infection, beyond the typical symptoms of fever, cough and dyspnea, the neuropsychiatric manifestations are added, which would be responsible for the symptoms of apathy, anorexia and muscular pain. These symptoms can go as far as the mental confusion, agitation, and psychosis that many patients may manifest even in initial stages, together with characteristic laboratory findings and pulmonary abnormalities [28].

Neurological complications

Today we know from the study of Mao L, et al. [25] that from the beginning of the disease, $66 \%$ of hospitalized patients started with the most common symptoms like fever, dry cough, and dyspnea, but 34 started with neuropsychiatric symptoms such as dizziness (16\%), headache (13\%), dysgeusia (6\%), and anosmia (5\%). $40 \%$ of the total studied patients were severe, and 60\% were non-severe, and within severe cases, 50\% had neuropsychiatric symptoms. Within the non-severe group, $30 \%$ of cases presented neuropsychiatric symptoms. While within the group of severe cases with neuropsychiatric symptoms, these were due to stroke (5.7\%), ischemic/haemorrhagic (4 to 1), altered consciousness (14.8\%), and rhabdomyolysis (19.3\%). Severe patients had a higher inflammatory response, including leucocytosis, neutrophilia, lymphopenia, increased CRP and higher D-dimer compared to non-severe cases, with $\mathrm{D}$-dimer being an indicator of consumption coagulation pattern. Furthermore, severe patients had multiple organ involvement, such as liver failure (increased LDH, T GO, T GP), kidney failure (increased urea and creatinine), and rhabdomyolysis (increased CPK levels). In the laboratory findings of patients with more severe neuropsychiatric symptoms, lymphopenia, platelet count, and increased urea were found. For the non-severe subgroup, there were no 
significant differences in the laboratory findings of patients with and without neuropsychiatric symptoms. Patients with rhabdomyolysis had leucocytosis, lymphopenia, and increased CRP and D-dimer. Abnormalities were a manifestation of increased inflammatory response and impaired coagulation function. Furthermore, it was observed that patients with rhabdomyolysis had multiple organ damage, including liver failure and kidney failure. For the severe group, patients with rhabdomyolysis had an increased inflammatory response (lymphopenia and increased CRP), liver failure, kidney, and rhabdomyolysis with increased CPK [25].

Likewise, 33\% of the total of patients, severe and non-severe, had diverse neuropsychiatric manifestations. The severe patients were older (58 \pm 10 years). The most frequent comorbidity in these cases was hypertension and, less frequently, they presented typical symptoms such as fever, cough and dyspnea. Furthermore, they were more likely to develop neuropsychiatric symptoms, especially mental confusion, agitation, and psychosis, and also acute cerebrovascular disease [25]..

$30 \%$ of the total of hospitalized patients had neuropsychiatric manifestations from the beginning of the infection [25], and the most severe or terminal patients probably develop neuropsychiatric symptoms in $45 \%$ as a manifestation of metabolic alteration or brain injury.

The interpretation of the data now is that, compared to non-severe patients with COVID19 , severe patients very frequently presented as predictors of mortality, elevated ferritin and IL- 6 as biomarkers of viral inflammation and neuropsychiatric symptoms as alteration of consciousness, mental confusion, agitation, cerebrovascular disease, encephalitis, and rhabdomyolysis.

\section{Discussion}

For all of the above, the new clinical information on COVID-19 would help to take into account the participation of neuropsychiatric manifestations, especially in patients with severe COVID-19, due to the rapid clinical deterioration or worsening that could be more related frequently with the appearance of mental confusion, confusional syndrome, agitation and strokes. Increasing the mortality rate. Likewise, during the epidemic period of COVID-19, when treating patients with neuropsychiatric manifestations, SARS-CoV-2 infection should be considered as a first-line differential diagnosis to avoid late or misdiagnosis and to prevent transmission [25].

Neuropsychiatric symptoms were the main form of manifestation of altered brain metabolism or neurological injury in reported COVID-19 patients. The pathophysiological mechanism could be the invasion of the central nervous system (CNS) by SARS-CoV-2, similar to the SARS and MERS viruses. Like other respiratory viruses, SARS-COV-2 can 
enter the CNS via the hematog enous or retrog rade neuronal pathway (since many patients presented anosmia or dysgeusia in initial stages). Lymphopenia has also been detected in patients with neuropsychiatric symptoms. This phenomenon may be indicative of immunosuppression in COVID-19 patients with neuropsychiatric symptoms, especially in severe subgroup.

On the other hand, severe cases have also been found to have high D-dimer suggestive of a hypercoagulable state. This may be the reason why seriously ill patients are more likely to develop cerebrovascular disease. In summary, SARS-CoV-2 can infect the nervous system, the skeletal muscle and the respiratory tract. In those with severe infection, neuropsychiatric involvement is more likely, including altered consciousness, mental confusion, agitation, acute cerebrovascular disease, and rhabdomyolysis. Nervous system involvement leads to poor prognosis due to greater hemodynamic instability, worsening clinical conditions and increased mortality. Therefore, for patients with COVID19, neuropsychiatric status should be monitored in addition to respiratory symptoms $[12,13,29,30]$.

\section{References}

[1] Liu TB, Chen XY, Miao GD, Zhang L, Zhang Q, Cheung T, et al. Recommendations on diagnostic criteria and prevention of SARS-related mental disorders. J Clin Psychol Med 2003;13:188-91.

[2] Mason BW, Lyons RA. Acute psychological effects of suspected bioterrorism. J Epidemiol Community Health 2003;57:353-4. https://doi.org/10.1136/jech.57.5.353. [3] Gale SD, Berrett AN, Erickson LD, Brown BL, Hedges DW. Association between virus exposure and depression in US adults. Psychiatry Res 2018;261:73-9. https://doi.org/10.1016/j.psychres.2017.12.037.

[4] Van Den Heuvel L, Chishinga N, Kinyanda E, Weiss H, Patel V, Ayles H, et al. Frequency and correlates of anxiety and mood disorders among TB- and HIV-infected Zambians. AIDS Care - Psychol Socio-Medical Asp AIDS/HIV 2013;25:1527-35. https://doi.org/10.1080/09540121.2013.793263.

[5] Kuan V, Denaxas S, Gonzalez-Izquierdo A, Direk K, Bhatti O, Husain S, et al. A chronological map of 308 physical and mental health conditions from 4 million individuals in the Eng lish National Health Service. Lancet Digit Heal 2019;1:e63-77. https://doi.org/10.1016/S2589-7500(19)30012-3. [6] Shultz JM, Cooper JL, Baing ana F, Oquendo MA, Espinel Z, Althouse BM, et al. The Role of Fear-Related Behaviors in the 2013-2016 West Africa Ebola Virus Disease 
Outbreak. Curr Psychiatry Rep 2016;18. https://doi.org/10.1007/s11920-016-0741-y.

[7] Person B, Sy F, Holton K, Govert B, Liang A, Garza B, et al. Fear and Stig ma: The Epidemic within the SARS Outbreak. Emerg Infect Dis 2004;10:358-63.

https://doi.org/10.3201/eid1002.030750.

[8] Mak IWC, Chu CM, Pan PC, Yiu MGC, Chan VL. Long-term psychiatric morbidities among SARS survivors. Gen Hosp Psychiatry 2009;31:318-26.

https://doi.org/10.1016/j.genhosppsych.2009.03.001.

[9] Gardner PJ, Moallef P. Psychological impact on SARS survivors: Critical review of the eng lish language literature. Can Psychol 2015;56:123-35.

https://doi.org/10.1037/a0037973.

[10] Blakey SM, Kirby AC, McClure KE, Elbogen EB, Beckham JC, Watkins LL, et al. Posttraumatic Safety Behaviors: Characteristics and Associations With Symptom Severity in Two Samples. Traumatology (T allahass Fla) 2019.

https://doi.org/10.1037/trm0000205.

[11] Arabi YM, Balkhy HH, Hayden FG, Bouchama A, Luke T, Baillie JK, et al. Middle east respiratory syndrome. N EngIJ Med 2017;376:584-94.

https://doi.org/10.1056/NEJMsr1408795.

[12] Hamming I, Timens W, Bulthuis MLC, Lely AT, Navis GJ, van Goor H. T issue distribution of ACE2 protein, the functional receptor for SARS coronavirus. A first step in understanding SARS pathogenesis. J Pathol 2004;203:631-7.

https://doi.org/10.1002/path.1570.

[13] CW, S Z, Y C, M Z. Single-cell RNA expression profiling of ACE2, the putative receptor of Wuhan 2019-nCoV, in the nasal tissue 2020.

https://doi.org/10.1101/2020.02.11.20022228.

[14] WHO. Middle East respiratory syndrome coronavirus (MERS-CoV). Web World Heal Organ 2020. https://www.who.int/emergencies/mers-cov/en/ (accessed 18 June 2020).

[15] Shigemura J, Ursano RJ, Morganstein JC, Kurosawa M, Benedek DM. Public responses to the novel 2019 coronavirus (2019-nCoV) in Japan: Mental health consequences and target populations. Psychiatry Clin Neurosci 2020;74:281-2. https://doi.org/10.1111/pcn.12988.

[16] Rubin G], Wessely S. The psychological effects of quarantining a city. BMJ 2020;368. https://doi.org/10.1136/bmj.m313.

[17] WHO. Mental health and psychosocial considerations during the COVID-19 outbreak. Web World Heal Organ 2020. https://www.who.int/docs/defaultsource/coronaviruse/mental-health-considerations.pdf (accessed 19 June 2020). [18] Xiang YT, Yang Y, Li W, Zhang L, Zhang Q, Cheung T, et al. Timely mental health care for the 2019 novel coronavirus outbreak is urgently needed. The Lancet Psychiatry 
2020;7:228-9. https://doi.org/10.1016/S2215-0366(20)30046-8.

[19] Yi Y, Lagniton PNP, Ye S, Li E, Xu RH. COVID-19: What has been learned and to be learned about the novel coronavirus disease. Int J Biol Sci 2020;16:1753-66. https://doi.org/10.7150/ijbs.45134.

[20] Wu P, Fang Y, Guan Z, Fan B, Kong J, Yao Z, et al. The psychological impact of the SARS epidemic on hospital employees in China: Exposure, risk perception, and altruistic acceptance of risk. Can J Psychiatry 2009;54:302-11. https://doi.org/10.1177/070674370905400504.

[21] Framing risk, reducing panic during virus outbreak - Asia Times n.d. https://asiatimes.com/2020/02/framing-risk-reducing-panic-during-virus-outbreak/ (accessed 19 June 2020).

[22] S F, S G. Promoting Psychological Well-Being in the Face of Serious Illness: When Theory, Research and Practice Inform Each Other. Psychooncology 2000;9. https://doi.org/10.1002/(SICI)1099-1611(200001/02)9:1<11::AID-PON424>3.0.CO;2-Z. [23] Soofi M, Najafi F, Karami-Matin B. Using Insights from Behavioral Economics to Mitigate the Spread of COVID-19. Appl Health Econ Health Policy 2020;18:345-50. https://doi.org/10.1007/s40258-020-00595-4.

[24] Williams MT, Mugno B, Franklin M, Faber S. Symptom dimensions in obsessivecompulsive disorder: Phenomenology and treatment outcomes with exposure and ritual prevention. Psychopathology 2013;46:365-76. https://doi.org/10.1159/000348582.

[25] Mao L, Jin H, Wang M, Hu Y, Chen S, He Q, et al. Neurologic Manifestations of Hospitalized Patients with Coronavirus Disease 2019 in Wuhan, China. JAMA Neurol 2020. https://doi.org/10.1001/jamaneurol.2020.1127.

[26] Ahmed SF, Quadeer AA, McKay MR. Preliminary Identification of Potential Vaccine Targets for the COVID-19 Coronavirus (SARS-CoV-2) Based on SARS-CoV Immunological Studies. Viruses 2020;12:254. https://doi.org/10.3390/v12030254.

[27] Mehta P, McAuley DF, Brown M, Sanchez E, Tattersall RS, Manson لע. COVID-19: consider cytokine storm syndromes and immunosuppression. Lancet 2020;395:1033-4. https://doi.org/10.1016/S0140-6736(20)30628-0.

[28] Guan W, Ni Z, Hu Y, Liang W, Ou C, He J, et al. Clinical characteristics of 2019 novel coronavirus infection in China. N EngIJ Med 2020:2020.02.06.20020974. https://doi.org/10.1101/2020.02.06.20020974.

[29] Ding Y, He L, Zhang Q, Huang Z, Che X, Hou J, et al. Organ distribution of severe acute respiratory syndrome (SARS) associated coronavirus (SARS-CoV) in SARS patients: Implications for pathogenesis virus transmission pathways. J Pathol 2004;203:622-30. https://doi.org/10.1002/path.1560.

[30] Cabello-Verrugio C, Morales MG, Rivera JC, Cabrera D, Simon F. Renin-angiotensin 
system: An old player with novel functions in skeletal muscle. Med Res Rev 2015;35:43763. https://doi.org/10.1002/med.21343. 